\title{
Article \\ Dysprosium Substituted Ce:YIG Thin Films for Temperature Insensitive Integrated Optical Isolator Applications
}

\author{
Zixuan Wei, Wei Yan *, Jun Qin, Longjiang Deng and Lei Bi *
}

\author{
National Engineering Research Center of Electromagnetic Radiation Control Materials, University of Electronic \\ Science and Technology of China, Chengdu 610054, China; zxwei@std.uestc.edu.cn (Z.W.); \\ qinjun@uestc.edu.cn (J.Q.); denglj@uestc.edu.cn (L.D.) \\ * Correspondence: weiyan@std.uestc.edu.cn (W.Y.); bilei@uestc.edu.cn (L.B.)
}

\begin{abstract}
Magneto-optical isolators are key components in photonic systems. Despite the progress of silicon-integrated optical isolators, the Faraday rotation of silicon-integrated magneto-optical materials, such as cerium-doped yttrium iron garnet (Ce:YIG), show a strong temperature dependence, limiting the temperature range for integrated nonreciprocal photonic device applications. In this work, we report dysprosium substituted Ce:YIG thin films ( $\mathrm{Dy}_{2} \mathrm{Ce}_{1} \mathrm{Fe}_{5} \mathrm{O}_{12}$, Dy:CeIG) showing a low temperature coefficient of Faraday rotation. A temperature insensitive range of the Faraday rotation is observed in between $25{ }^{\circ} \mathrm{C}$ to $70{ }^{\circ} \mathrm{C}$ for this material, compared to $20 \%$ variation of the Faraday rotation in Ce:YIG thin films. A Dy:CeIG based temperature insensitive silicon-integrated optical isolator operating in the temperature range of $23{ }^{\circ} \mathrm{C}$ to $70^{\circ} \mathrm{C}$ is experimentally demonstrated.
\end{abstract}

Keywords: magneto-optical materials; faraday rotation; temperature insensitivity

check for updates

Citation: Wei, Z.; Yan, W.; Qin, J.; Deng, L.; Bi, L. Dysprosium Substituted Ce:YIG Thin Films for Temperature Insensitive Integrated Optical Isolator Applications. Materials 2022, 15, 1691. https:// doi.org/10.3390/ma15051691

Academic Editor: Mikhael Bechelany

Received: 29 January 2022

Accepted: 17 February 2022

Published: 24 February 2022

Publisher's Note: MDPI stays neutral with regard to jurisdictional claims in published maps and institutional affiliations.

Copyright: (C) 2022 by the authors. Licensee MDPI, Basel, Switzerland. This article is an open access article distributed under the terms and conditions of the Creative Commons Attribution (CC BY) license (https:// creativecommons.org/licenses/by/ $4.0 /)$.

\section{Introduction}

Integrated magneto-optical (MO) isolators and circulators are key components for silicon-integrated photonic circuits (PICs) [1-3]. At present, rare earth-doped yttrium iron garnet (RIG) is the most widely-used magneto-optical material in integrated MO devices. In the past decade, significant progress has been achieved in bonding or deposition of MO garnet thin films on silicon, such as cerium-doped yttrium iron garnet thin films (Ce:YIG). Silicon-integrated MO nonreciprocal photonic devices including optical isolators [4-6], circulators [1,7], modulators [8], and optical switches [9] have been developed, showing a high isolation ratio, low insertion loss, and fast switching/modulation speed. For practical applications, on-shelf bulk optical isolators or circulators using bismuth-doped rare earth iron garnet materials usually show a high performance in the temperature range from $-20{ }^{\circ} \mathrm{C}$ to $70^{\circ} \mathrm{C}$. However, Ce:YIG show a stronger temperature dependence of the Faraday rotation, leading to large drift of the operation wavelength as a function of temperature [10,11]. This problem is particularly prominent for phase-sensitive devices such as the Mach-Zehnder interferometer (MZI) based optical isolators, which influences both the isolation ratio and insertion loss, resulting in a narrow operation temperature range.

Several previous works were carried out to address the temperature stability problem of Ce:YIG. Enno et al. studied the Faraday rotation angle of epitaxial Ce:YIG thin films from $-175{ }^{\circ} \mathrm{C}$ to $+25^{\circ} \mathrm{C}$ [12]. The Faraday rotation monotonically decrease with increasing temperature. The Faraday rotation shows temperature dependence of about $-20 \mathrm{deg} \cdot \mathrm{cm}^{-1} \cdot \mathrm{K}^{-1}$ in this temperature range. Huang et al. characterized silicon-integrated ring resonator optical isolators based on bonded Ce:YIG epitaxial thin films. They observed that the nonreciprocal-phase shift (NRPS) effect of Ce:YIG decreases when the material is heated up by an integrated electromagnet, which results in a reduced bandwidth and isolation ratio [13]. To solve this problem, Furuya et al. designed MZI optical isolators with a special designed length of the reciprocal phase shift (RPS) waveguide. The temperature dependence of the RPS and NRPS waveguide cancels out, results in an almost temperature 
insensitive transmission spectrum for the backward propagation direction in a temperature range of $20-60{ }^{\circ} \mathrm{C}$ [10]. However, the device still suffers from temperature dependence for forward propagation, leading to higher insertion loss when temperature is changed. A similar result is also observed in SiN-based optical isolators reported in our previous work [11]. Due to the low thermo-optic coefficient of $\mathrm{SiN}$, the isolators maintained a relatively stable operation wavelength, showing a small wavelength drift within $4 \mathrm{~nm}$ in a temperature range of $20-70{ }^{\circ} \mathrm{C}$. However, the device still shows a deteriorated performance due to the temperature dependence of the $\mathrm{MO}$ effect. Fundamentally, these problems originated from the temperature dependence of the Faraday effect of the MO material, which cannot be fully resolved by the device design. Therefore, exploring new $\mathrm{MO}$ materials with a higher temperature stability is important for solving temperature stability issues.

In this paper, we report a new MO material system: Dysprosium-substituted Ce:YIG (Dy:CeIG), showing a lower temperature dependence of the Faraday rotation compared to Ce:YIG films. Thanks to the negative temperature coefficient of the Faraday rotation of the $\mathrm{Dy}_{3} \mathrm{Fe}_{5} \mathrm{O}_{12}$ material [14-16], the $\mathrm{Dy}_{2} \mathrm{Ce}_{1} \mathrm{Fe}_{5} \mathrm{O}_{12}$ deposited on silicon shows a flat temperature dependence plateau with less than $\pm 5 \%$ Faraday rotation change in the temperature range from $20{ }^{\circ} \mathrm{C}$ to $70{ }^{\circ} \mathrm{C}$, in contrast to the almost $20 \%$ decrease of the Faraday rotation of $\mathrm{Ce}: \mathrm{YIG}$ of the same temperature range. We also fabricated integrated MZI isolators on silicon using both materials, demonstrating higher stability of the Dy:CeIG based device. The good temperature stability of Dy:CeIG makes it a promising material for silicon-integrated nonreciprocal photonic device applications.

\section{Materials and Methods}

\subsection{Material Fabrication and Characterization}

Using the two-step deposition method of our previous study, we deposited $100 \mathrm{~nm}$ of thick Ce:YIG with $50 \mathrm{~nm}$ of thick YIG and $100 \mathrm{~nm}$ of thick Dy:CeIG with $50 \mathrm{~nm}$ of thick YIG, respectively, on (100) Si by pulsed laser deposition (PLD) [2,17]. Then the films were subsequently rapid thermal annealed at $850{ }^{\circ} \mathrm{C}$ under an oxygen partial pressure of 2 Torr for $3 \mathrm{~min}$. The crystal structure was characterized by X-ray diffraction (Rigaku Ultima IV) with a $\mathrm{Cu}-\mathrm{K}_{\alpha}$ radiation source. The XRD patterns were collected over the $2 \theta$ range of $25-40^{\circ}$ with a step size of $0.02^{\circ}$. Room-temperature in-plane and out-of-plane magnetic hysteresis loops were measured by vibrating sample magnetometry (VSM). The MO hysteresis loops were measured by using a custom-built Faraday effect characterization system with field and light propagation directions out of plane [17]. The maximum of the magnetic field is $4800 \mathrm{Gs}$. The sample holder of the Faraday effect characterization system is a tunable heater which has a heating range from room temperature (RT) to $120^{\circ} \mathrm{C}$.

\subsection{Device Preparation and Characterization}

Use the same methods in Section 2.1, $86 \mathrm{~nm}$ of Ce:YIG with $50 \mathrm{~nm}$ of YIG thin films and $180 \mathrm{~nm}$ of Dy:CeIG with $50 \mathrm{~nm}$ of YIG-thin films were deposited, respectively, on the top of $\mathrm{SiN}$ waveguides as discussed in [11]. The devices were characterized on a polarization-maintaining, fiber-butt-coupled system. The transmission spectrums were obtained from the same setup of our previous work [11]. An in-plane magnetic field (1000 Gs) was applied perpendicular to the propagation direction of light to saturate the magnetization of MO-thin films. Forward and backward transmittance between the input and output ports is given by [6]:

$$
T_{\text {for, back }}=\cos ^{2}\left(\frac{\theta_{R P S} \mp \theta_{N R P S}}{2}\right)
$$

where $\theta_{R P S}$ is the reciprocal phase shift (RPS) of the SiN waveguides, and $\theta_{N R P S}$ is the nonreciprocal phase shift (NRPS) of the $\mathrm{MO} / \mathrm{SiN}$ waveguides, which is linearly related to the Faraday rotation $\theta_{F}[7,18]$ : 


$$
\theta_{N R P S}=\Delta \beta \cdot L_{N R P S}=L_{N R P S} \cdot \frac{2 \beta}{\omega \varepsilon_{0} N} \iint \frac{\lambda \theta_{F}}{\pi n_{0}^{3}} H_{x} \partial_{y} H_{x} \mathrm{~d} x \mathrm{~d} y
$$

where $\omega$ is the angular frequency, $\Delta \beta$ is the propagation constants difference between forward and backward propagation light, $\beta$ is the propagation of the fundamental TM mode, $N$ is the power flux along the $\mathrm{z}$ direction, $n_{0}$ is the refractive index of the MO material [7], and $H_{x}$ is the magnetic field along the $x$ direction.

From the measured NRPS, the Faraday rotation of the MO films can be calculated using the following equations:

$$
\frac{\Theta_{f}(T)}{\Theta_{f}^{R T}}=\frac{\theta_{N R P S}(T)}{\theta_{N R P S}^{R T}}
$$

where $\Theta_{f}^{R T}$ is the Faraday rotation angle of MO films at room temperature. $\theta_{N R P S}^{R T}$ is the NRPS at room temperature.

We consider the Faraday rotation is dispersionless in the measured wavelength range, which is a good approximation based on previous measurements [17]. Both devices are characterized under a temperature range from $23^{\circ} \mathrm{C}(\mathrm{RT})$ to $100{ }^{\circ} \mathrm{C}$, with a step size of $10{ }^{\circ} \mathrm{C}$.

\section{Results and Discussion}

\subsection{Material Characterization}

Figure 1a shows the X-ray diffraction patterns of Dy:CeIG and Ce:YIG thin films. Both films show well-crystallized diffraction peaks of the polycrystalline garnet phase. The peak at $2 \theta=33.1^{\circ}$ originated from the (200) planes of the silicon substrate. Due to the ionic radius of $\mathrm{Dy}^{3+}$ ions $\left(1.083 \AA\right.$ ) being larger than $\mathrm{Y}^{3+}$ ions $(1.02 \AA)$, the lattice constant of Dy:CeIG is larger than that of Ce:YIG. The calculated lattice constants of Dy:CeIG and Ce:YIG are $12.42 \AA$ and $12.37 \AA$, respectively, consistent with previous reports [17]. Figure $1 \mathrm{~b}$ shows the room temperature ( $300 \mathrm{~K})$ magnetic hysteresis loops of Dy:CeIG. The films show a similar saturation magnetization field for in-plane and out-of-plane magnetization directions, which is attributed to the competition between magnetoelastic anisotropy and shape anisotropy [17]. The saturation magnetization of Dy:CeIG thin film is $111 \mathrm{emu} / \mathrm{cm}^{3}$, comparable to our previous report [17].

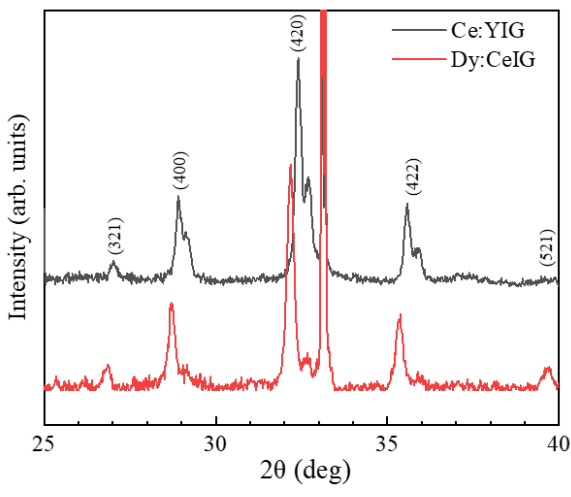

(a)

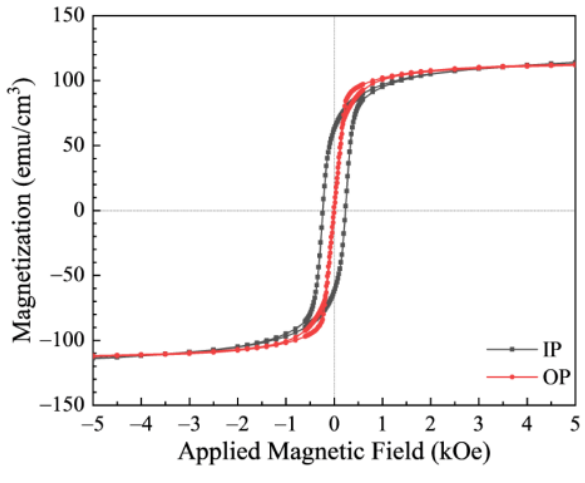

(b)

Figure 1. (a) X-ray diffraction patterns of Dy:CeIG and Ce:YIG thin films with a bottom yttrium iron garnet (YIG) seed layer of $50 \mathrm{~nm}$ in a $2 \theta$ range from $25^{\circ}$ to $40^{\circ}$. (b) Room temperature in-plane and out-of-plane magnetization hysteresis loops for Dy:CeIG thin films.

Next, we characterized the Faraday rotation hysteresis loops of Dy:CeIG and Ce:YIG thin films in the temperature range of $25^{\circ} \mathrm{C}$ to $120^{\circ} \mathrm{C}$, as shown in Figure $2 \mathrm{a}$, b. We used the wavelength of $1310 \mathrm{~nm}$ for higher Faraday rotation angles and better visibility of the 
temperature dependence. The temperature dependence of the Faraday rotation can be quantified by the temperature coefficient:

$$
\xi=\frac{1}{\theta_{f}} \cdot\left(\frac{d \theta_{f}}{d T}\right)_{T}\left(c m^{-1} \cdot K^{-1}\right) .
$$

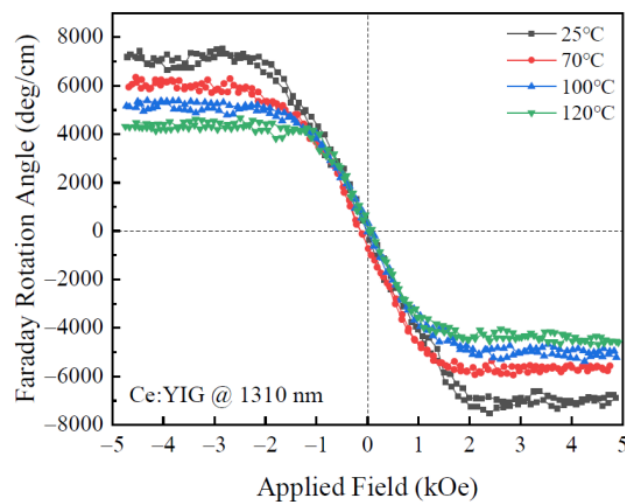

(a)

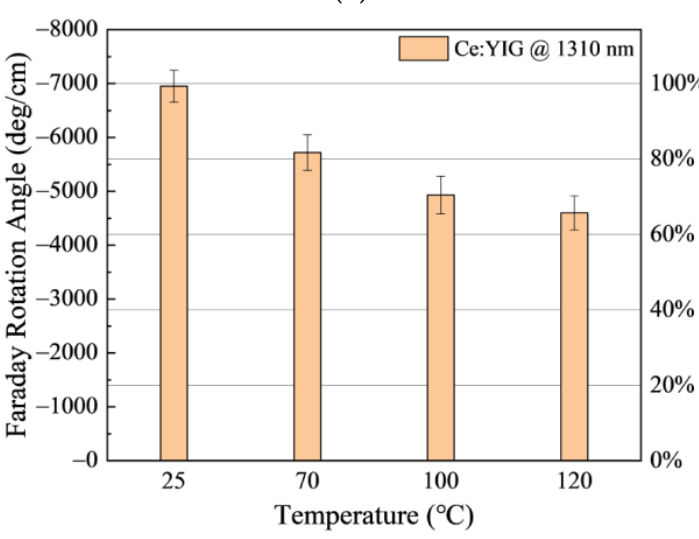

(c)

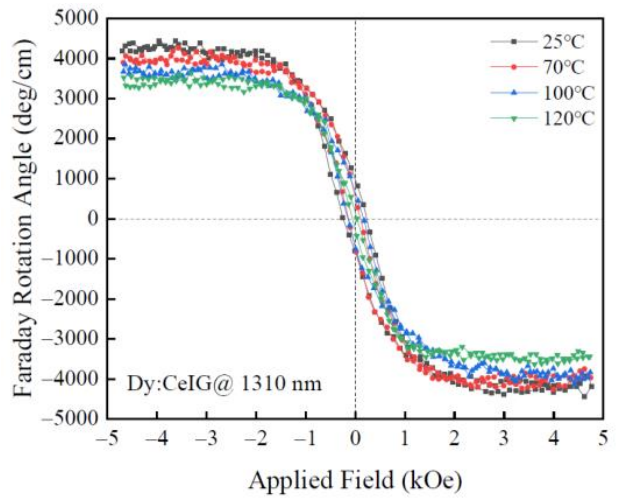

(b)

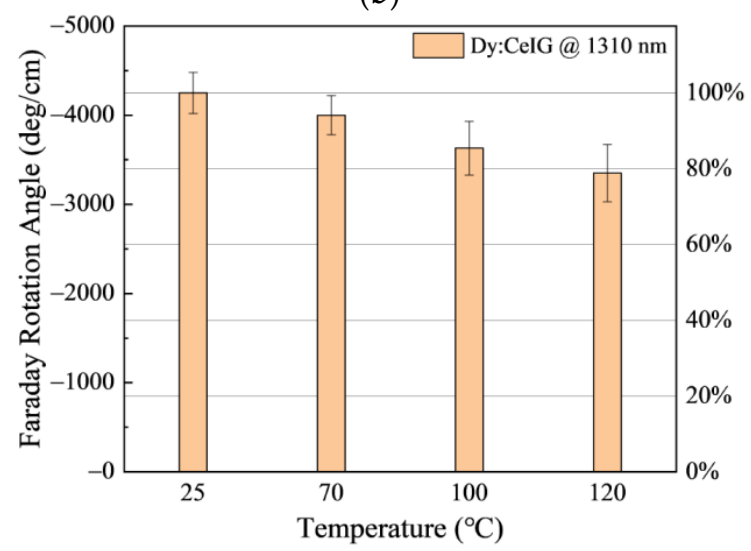

(d)

Figure 2. Temperature dependence of the Faraday rotation (FR) hysteresis loops at $1310 \mathrm{~nm}$ wavelength of (a) Ce:YIG and (b) Dy:CeIG thin films on silicon. Curves of different colors indicate different temperatures. Faraday rotation angle of both films as functions of temperature: (c) Ce:YIG and (d) Dy:CeIG. The error bar is the standard deviation from three consecutive measurements at the same temperature.

For Ce:YIG, the saturation Faraday rotation angle reaches $-6950 \mathrm{deg} / \mathrm{cm}$ at $25^{\circ} \mathrm{C}$. As the temperature rises, this value declines to $-5730 \mathrm{deg} / \mathrm{cm}$ at $70{ }^{\circ} \mathrm{C}$ and $-4500 \mathrm{deg} / \mathrm{cm}$ at $120^{\circ} \mathrm{C}$, showing a monotonic decrease in the measured temperature range, with a temperature coefficient of $3.7 \times 10^{-3} \mathrm{~cm}^{-1} \cdot \mathrm{K}^{-1}$. Whereas for Dy:CeIG, a lower temperature dependence of the Faraday rotation is observed. For the temperature range of $25^{\circ} \mathrm{C}$ to $70{ }^{\circ} \mathrm{C}$, the Faraday rotation angle of Dy:CeIG thin film decreases from $-4250 \mathrm{deg} / \mathrm{cm}$ to $-3800 \mathrm{deg} / \mathrm{cm}$. When the temperature continues to rise, the Faraday rotation of the film decreases to $-3200 \mathrm{deg} / \mathrm{cm}$ at $120{ }^{\circ} \mathrm{C}$. A lower temperature coefficient of $2.6 \times 10^{-3} \mathrm{deg} \cdot \mathrm{cm}^{-1} \cdot \mathrm{K}^{-1}$ is demonstrated in Dy:CeIG. The Faraday rotation value normalized to a room temperature value is summarized in Figure 2c,d for both materials. The error bar represents the standard deviation from three consecutive measurements at the same temperature. We notice the Faraday rotation of Ce:YIG decreases to almost $65 \%$ at $120{ }^{\circ} \mathrm{C}$ compared to its room temperature value, whereas for Dy:CeIG, it decreases to about $80 \%$. In particular, for the temperature range of $70^{\circ} \mathrm{C}$ and lower, which is the typical application temperature range for optical isolators, Dy:CeIG shows a much higher temperature stability compared to Ce:YIG. 


\subsection{Device Characterization}

We further fabricated MZI isolator devices to measure the temperature stability of Dy:CeIG and Ce:YIG films [11]. Figure 3a,b shows the device structure. Details of the device design and fabrication can be found in our previous reports [11].

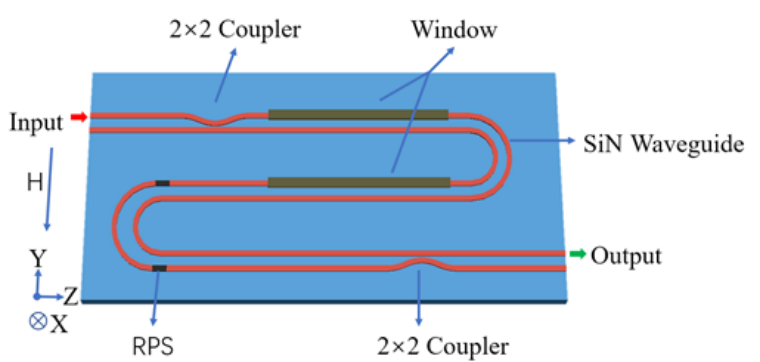

(a)

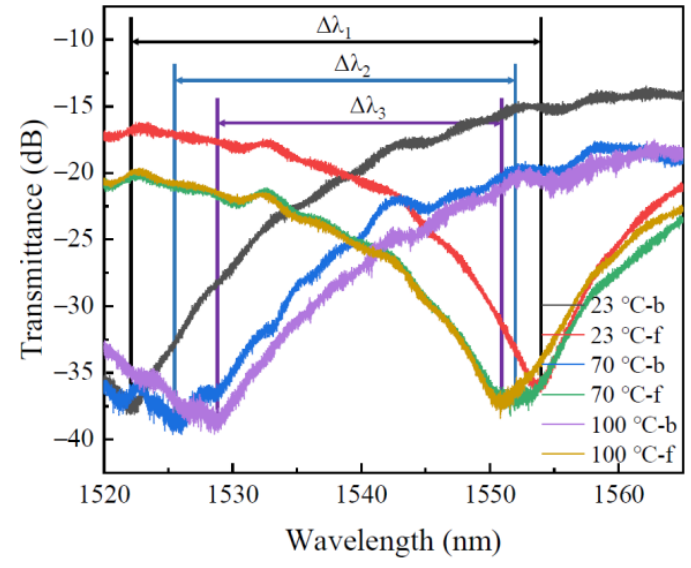

(c)

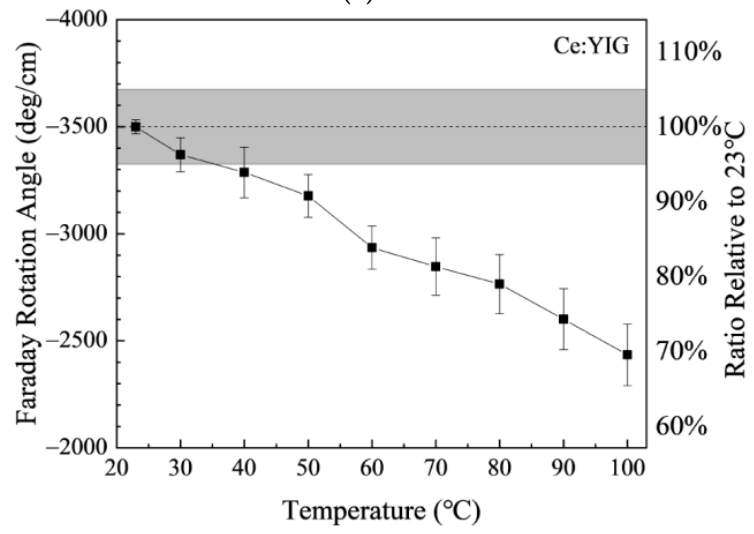

(e)

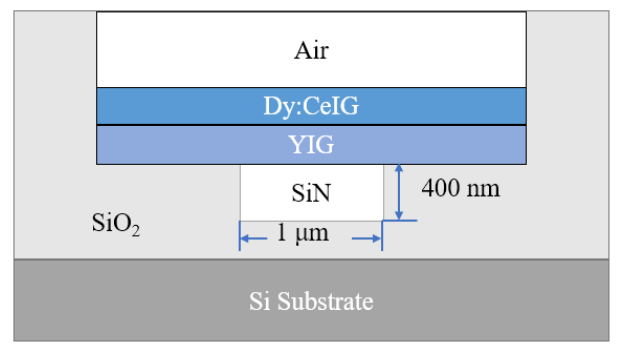

(b)

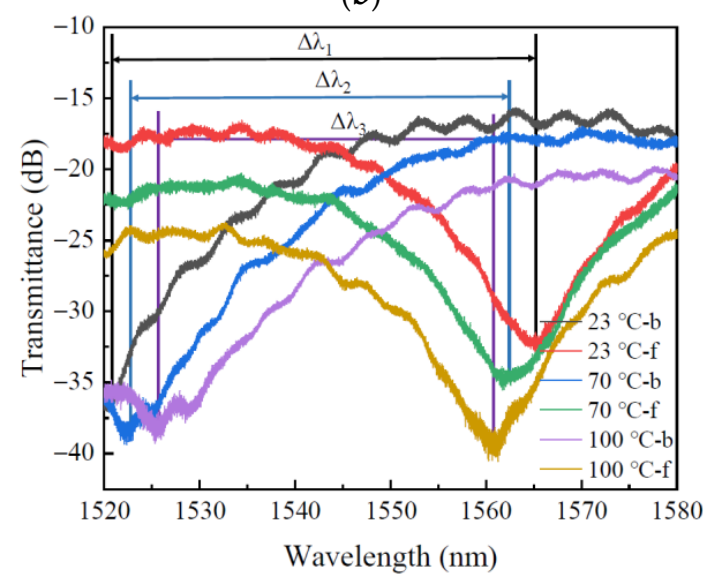

(d)

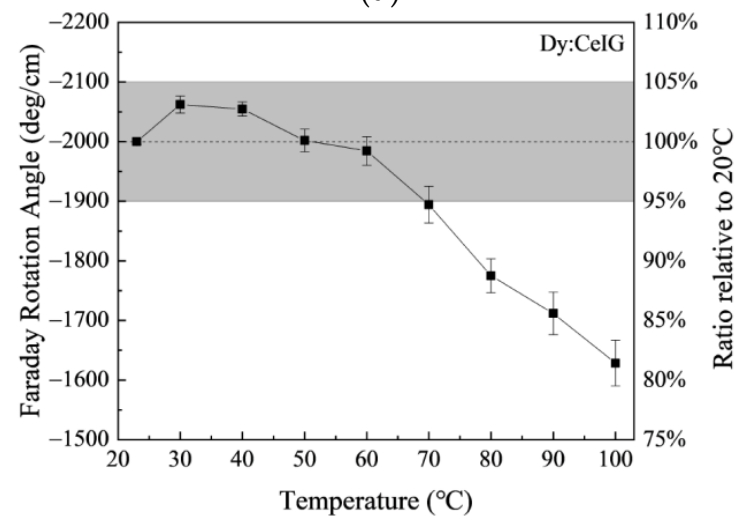

(f)

Figure 3. (a) Schematics of integrated magneto-optical isolators on $\mathrm{SiN}$. (b) Cross section of MO/SiN waveguide. Transmission spectra of SiN optical isolators under different device temperatures of $23^{\circ} \mathrm{C}, 80{ }^{\circ} \mathrm{C}$, and $100{ }^{\circ} \mathrm{C}$, respectively for (c) Ce:YIG and (d) Dy:CeIG. " $\mathrm{f}$ " and " $\mathrm{b}$ " correspond to forward and backward propagation light. Faraday rotation angle versus temperature resulting from transmission spectra, respectively for (e) Ce:YIG and (f) Dy:CeIG. The error bar is the standard deviation from three consecutive measurements of the transmission spectrum.

Figure 3c,d shows the transmission spectra of the TM-mode SiN optical isolator with Ce:YIG and Dy:CeIG as the MO materials. Clear one-way transmission is observed on both devices in the measurement temperature range. The forward and backward propagation spectra moved close to each other with an increasing temperature due to 
the lower NRPS and Faraday effects. For the Ce:YIG sample, the spectra shifted for $\Delta \lambda_{1}-\Delta \lambda_{2}=5.963 \mathrm{~nm}$ from $23^{\circ} \mathrm{C}$ to $70^{\circ} \mathrm{C}$, as shown in Figure 3c. They further shifted for $\Delta \lambda_{1}-\Delta \lambda_{3}=9.732 \mathrm{~nm}$ from $70^{\circ} \mathrm{C}$ to $100{ }^{\circ} \mathrm{C}$. For the Dy:CeIG sample, the spectra showed less wavelength drift compared to Ce:YIG, which is $\Delta \lambda_{1}-\Delta \lambda_{2}=2.218 \mathrm{~nm}$ for the temperature range from $23{ }^{\circ} \mathrm{C}$ to $70{ }^{\circ} \mathrm{C}$ and $\Delta \lambda_{1}-\Delta \lambda_{3}=7.788 \mathrm{~nm}$ for the temperature range from $70{ }^{\circ} \mathrm{C}$ to $100{ }^{\circ} \mathrm{C}$, respectively. Therefore the Dy:CeIG sample shows better temperature stability compared to Ce:YIG, especially for temperatures below $70{ }^{\circ} \mathrm{C}$. Based on the transmission spectra of the devices at different temperatures, we calculated the Faraday rotation angle of Ce:YIG and Dy:CeIG at each temperature following Equation (3). Figure 3e,f shows the calculated Faraday rotation angle versus temperature for both materials at the measured wavelength range. The room temperature Faraday rotation angle at the $1550 \mathrm{~nm}$ wavelength of Ce:YIG and Dy:CeIG are $-3500 \mathrm{deg} / \mathrm{cm}$ and $-2000 \mathrm{deg} / \mathrm{cm}$, respectively. For Ce:YIG, when the temperature increased from $23{ }^{\circ} \mathrm{C}$ to $100{ }^{\circ} \mathrm{C}$, the Faraday rotation angle decreased from $3500 \mathrm{deg} / \mathrm{cm}$ to $2348 \mathrm{deg} / \mathrm{cm}$, which was only $67 \%$ of the initial value, consistent with Figure 2c. For Dy:CeIG, the Faraday rotation angle increased to $-2050 \mathrm{deg} / \mathrm{cm}$ up to $40{ }^{\circ} \mathrm{C}$, then decreased to $-1890 \mathrm{deg} / \mathrm{cm}$ at $70{ }^{\circ} \mathrm{C}$, showing only a $5 \%$ change. The increase of the Faraday rotation below $40^{\circ} \mathrm{C}$ is possibly due to the increase of the magnetization of this material at this temperature range, considering a compensation temperature of $225 \mathrm{~K}$ in $\mathrm{Dy}_{3} \mathrm{Fe}_{5} \mathrm{O}_{12}[19,20]$. When the temperature continues to rise, the Faraday rotation angle of Dy:CeIG thin film decreased to $-1628 \mathrm{deg} / \mathrm{cm}$ at $100{ }^{\circ} \mathrm{C}$, which is $81.4 \%$ of the initial value. In terms of device performance, a $5 \%$ Faraday rotation variation leads to spectra shift of $2.5 \mathrm{~nm}$ for the current device structure. For Ce:YIG based devices, this spectra shift will take place as the temperature increases from $23^{\circ} \mathrm{C}$ to $35^{\circ} \mathrm{C}$, whereas this temperature range is up to $23^{\circ} \mathrm{C}$ to $100^{\circ} \mathrm{C}$ for Dy:CeIG. Our observation demonstrates that Dy:CeIG based integrated optical isolators show higher temperature stability in the temperature range of $23^{\circ} \mathrm{C}$ to $70^{\circ} \mathrm{C}$.

\section{Conclusions}

In summary, we demonstrated a temperature stable magneto-optical material $\mathrm{Dy}_{2} \mathrm{Ce}_{1} \mathrm{Fe}_{5} \mathrm{O}_{12}$ for silicon-integrated nonreciprocal photonic device applications. By replacing $\mathrm{Y}^{3+}$ ions with $\mathrm{Dy}^{3+}$ ions in Ce:YIG, we demonstrate less than a $\pm 5 \%$ variation of the thin film Faraday rotation from the temperature of $25^{\circ} \mathrm{C}$ to $70{ }^{\circ} \mathrm{C}$, compared to $20 \%$ in Ce:YIG. Integrated-MO isolators consist of Dy:CeIG thin films show better temperature stability compared to Ce:YIG based devices in the temperature range of $23{ }^{\circ} \mathrm{C}$ to $70{ }^{\circ} \mathrm{C}$, which is highly desired for practical applications.

Author Contributions: Conceptualization, Z.W. and W.Y.; methodology, L.B.; software, Z.W.; validation, Z.W., W.Y. and J.Q.; formal analysis, W.Y. and J.Q.; investigation, Z.W.; data curation, Z.W. and W.Y.; writing—original draft preparation, Z.W.; writing—review and editing, W.Y. and L.B.; supervision, L.B.; project administration, J.Q.; funding acquisition, L.D. and L.B. All authors have read and agreed to the published version of the manuscript.

Funding: This research was funded by the Ministry of Science and Technology of the People's Republic of China (MOST) (grant no. 2018YFE0109200), National Natural Science Foundation of China (NSFC) (grant nos. 51972044 and 52021001), Sichuan Provincial Science and Technology Department (grant no. 2019YFH0154), and the Fundamental Research Funds for the Central Universities (grant no. ZYGX2020J005).

Institutional Review Board Statement: Not applicable.

Informed Consent Statement: Not applicable.

Data Availability Statement: The data presented in this study are available from the corresponding authors upon reasonable request.

Conflicts of Interest: The authors declare no conflict of interest. 


\section{References}

1. Zhang, Y.; Du, Q.; Wang, C.; Fakhrul, T.; Liu, S.; Deng, L.; Huang, D.; Pintus, P.; Bowers, J.; Ross, C.A.; et al. Monolithic integration of broadband optical isolators for polarization-diverse silicon photonics. Optica 2019, 6, 473-478. [CrossRef]

2. Bi, L.; Hu, J.; Jiang, P.; Kim, D.H.; Dionne, G.F.; Kimerling, L.C.; Ross, C.A. On-chip optical isolation in monolithically integrated non-reciprocal optical resonators. Nat. Photonics 2011, 5, 758-762. [CrossRef]

3. Du, Q.; Wang, C.; Zhang, Y.; Zhang, Y.; Fakhrul, T.; Zhang, W.; Gonçalves, C.; Blanco, C.; Richardson, K.; Deng, L.; et al. Monolithic On-chip Magneto-optical Isolator with $3 \mathrm{~dB}$ Insertion Loss and $40 \mathrm{~dB}$ Isolation Ratio. ACS Photonics 2018, 5, 5010-5016. [CrossRef]

4. Zhang, C.; Dulal, P.; Stadler, B.J.H.; Hutchings, D.C. Monolithically-Integrated TE-mode 1D Silicon-on-Insulator Isolators using Seedlayer-Free Garnet. Sci. Rep. 2017, 7, 5820. [CrossRef] [PubMed]

5. Huang, D.; Pintus, P.; Shoji, Y.; Morton, P.; Mizumoto, T.; Bowers, J.E. Integrated broadband Ce:YIG/Si Mach-Zehnder optical isolators with over $100 \mathrm{~nm}$ tuning range. Opt. Lett. 2017, 42, 4901-4904. [CrossRef] [PubMed]

6. Shoji, Y.; Mizumoto, T. Silicon Waveguide Optical Isolator with Directly Bonded Magneto-Optical Garnet. Appl. Sci. 2019, 9, 609. [CrossRef]

7. Shoji, Y.; Miura, K.; Mizumoto, T. Optical nonreciprocal devices based on magneto-optical phase shift in silicon photonics. J. Opt. 2016, 18, 013001. [CrossRef]

8. Pintus, P.; Ranzani, L.; Pinna, S.; Huang, D.; Gustafsson, M.V.; Karinou, F.; Casula, G.A.; Shoji, Y.; Takamura, Y.; Mizumoto, T. A low-power integrated magneto-optic modulator on silicon for cryogenic applications. arXiv 2021, arXiv:2109.04538.

9. Murai, T.; Shoji, Y.; Nishiyama, N.; Mizumoto, T. Nonvolatile magneto-optical switches integrated with a magnet stripe array. Opt. Express 2020, 28, 31675-31685. [CrossRef]

10. Furuya, K.; Nemoto, T.; Kato, K.; Shoji, Y.; Mizumoto, T. Athermal Operation of a Waveguide Optical Isolator Based on Canceling Phase Deviations in a Mach-Zehnder Interferometer. J. Lightwave Technol. 2016, 34, 1699-1705. [CrossRef]

11. Yan, W.; Yang, Y.; Liu, S.; Zhang, Y.; Xia, S.; Kang, T.; Yang, W.; Qin, J.; Deng, L.; Bi, L. Waveguide-integrated high-performance magneto-optical isolators and circulators on silicon nitride platforms. Optica 2020, 7, 1555. [CrossRef]

12. Lage, E.; Beran, L.; Quindeau, A.U.; Ohnoutek, L.; Kucera, M.; Antos, R.; Sani, S.R.; Dionne, G.F.; Veis, M.; Ross, C.A. Temperaturedependent Faraday rotation and magnetization reorientation in cerium-substituted yttrium iron garnet thin films. APL Mater. 2017, 5, 036104. [CrossRef]

13. Huang, D.; Pintus, P.; Bowers, J.E. Towards heterogeneous integration of optical isolators and circulators with lasers on silicon [Invited]. Opt. Mater. Express 2018, 8, 2471-2483. [CrossRef]

14. Booth, R.C.; White, E.A.D. Magneto-optic properties of rare earth iron garnet crystals in the wavelength range $1.1-1.7 \mu \mathrm{m}$ and their use in device fabrication. J. Phys. D Appl. Phys. 1984, 17, 579-587. [CrossRef]

15. Umezawa, H.; Yokoyama, Y.; Koshizuka, N. Temperature dependence of Faraday rotation in Bi-substituted terbium iron garnet films. J. Appl. Phys. 1988, 63, 3113-3115. [CrossRef]

16. Zhang, X.W.; Zhang, S.Y.; Han, G.R. Growth and characterization of magneto-optical single-crystal ReYbBiIG with temperaturestabilized Faraday rotation. J. Magn. Magn. Mater. 2002, 246, 67-72. [CrossRef]

17. Zhang, Y.; Du, Q.; Wang, C.; Yan, W.; Deng, L.; Hu, J.; Ross, C.A.; Bi, L. Dysprosium substituted Ce:YIG thin films with perpendicular magnetic anisotropy for silicon integrated optical isolator applications. APL Mater. 2019, 7, 081119. [CrossRef]

18. Yokoi, H. Calculation of nonreciprocal phase shift in magneto-optic waveguides with Ce:YIG layer. Opt. Mater. 2008, 31, 189-192. [CrossRef]

19. Sayetat, F. Huge magnetostriction in $\mathrm{Tb}_{3} \mathrm{Fe}_{5} \mathrm{O}_{12}, \mathrm{Dy}_{3} \mathrm{Fe}_{5} \mathrm{O}_{12}, \mathrm{Ho}_{3} \mathrm{Fe}_{5} \mathrm{O}_{12}, \mathrm{Er}_{3} \mathrm{Fe}_{5} \mathrm{O}_{12}$ garnets. J. Magn. Magn. Mater. 1986, 58, 334-346. [CrossRef]

20. Ostorero, J.; Escorne, M.; Pecheron-Guegan, A.; Soulette, F.; Le Gall, H. Dy ${ }_{3} \mathrm{Fe}_{5} \mathrm{O}_{12}$ garnet thin films grown from sputtering of metallic targets. J. Appl. Phys. 1994, 75, 6103-6105. [CrossRef] 